\title{
MULTI-RETAILER SYSTEMS: PULL OR PUSH STRATEGY?
}

\section{Erik van der Sluis, University of Amsterdam, The Netherlands}

We consider stochastic multi-retailer systems under periodic review. Retailers are replenished by a central depot which in turn places orders at an outside supplier. Both retailers and depot incur fixed cost for placing order and holding cost for each unit stocked at the end of a period. Two rolling horizon procedures based on a pull or a push approach are examined. In the former retailers replenish independently and the decisions at the depot are based on the expected order schedule of the retailers. In the latter all replenishment decisions are taken simultaneously with the depot ordering either at constant intervals or at variable intervals. The objective of this paper is to point out which approach performs best in a stochastic system.

Simulation experiments showed that a straightforward implemented rolling horizon procedure can be improved by adjusting the order decision of the retailers and the depot. This resulted in time-dependent $\left(s, S_{\mathfrak{t}}\right)$-strategies for retailers in a push system. Cost and demand structure of the retailers may indicate which approach gives the best results. 